\title{
Tratamiento conjunto con mirtazapina y terapia antiretroviral para la leucoencefalopatía multifocal progresiva asociada a infección por VIH-1: presentación de un caso clínico y revisión de la literatura
}

\author{
Martín Lasso e Inés Cerón
}

\section{Mirtazapine and antiretroviral therapy in the treatment of progressive multifocal leukoencephalopathy associated with HIV-1 infection: report of a case and review of literature}

We report a 43 years old HIV-1 infected male who developed a severe subacute neurological damage because of a progressive multifocal leukoencephalopathy confirmed by PCR for JC virus. The patient was treated with antiretroviral therapy in adequate doses for CNS penetration and mirtazapine, an antidepressant inhibitor of serotonin receptors. His evolution during one year follow up has been favorable in both, clinically and images.

Key words: Progressive multifocal leukoencephalopathy, mirtazapine, HAART, JC virus.

Palabras clave: Leucoencefalopatia multifocal progresiva, mirtazapina, terapia anti-retroviral, virus JC.

\section{Introduccción}

\section{L}

a leucoencefalopatía multifocal progresiva (LEMP) es una infección oportunista causada por el virus JC, denominado así por las iniciales de John Cunningham, el paciente en quien se detectó por primera vez este agente en $1971^{1}$. Pertenece a la familia Papillomaviridae y su infección causa una enfermedad desmielinizante que compromete gravemente al sistema nervioso central (SNC). La infección primaria viral asintomática habitualmente ocurre en la niñez, permaneciendo en forma latente en los tejidos renal y linfático; en condiciones de inmunosupresión intensa se genera una reactivación viral que causa la enfermedad. En el contexto del paciente portador de VIH-1 representa una infección oportunista (IO) poco frecuente siendo su incidencia anual en la época previa a la llegada de la terapia anti retroviral (TARV) en grandes series europeas de un $0,9 \%$ y ha descendido a $0,1 \%$ en la actualidad con el uso de $\mathrm{TARV}^{2}$. En Chile representa 1,1\% de todas las enfermedades oportunistas (información no publicada Grupo SIDA Chile 2009). Sin embargo, junto con el linfoma no Hodgkin, es una de las enfermedades marcadoras de SIDA más graves y con más riesgo vital en pacientes vírgenes a tratamiento que inician $\mathrm{TARV}^{3}$, debido principalmente a su rápida progresión y ausencia de una terapia antiviral específica. En este contexto se presenta el siguiente caso clínico en el que la asociación de TARV y mirtazapina se relacionaron con la evolución favorable del paciente.

\section{Caso clínico}

Paciente de 43 años, homosexual, portador de VIH-1 con diagnóstico confirmado por el ISP en febrero de 2009. Tenía el antecedente de neumonía por $P$. jiroveci, candidiasis orofaríngea-esofágica e infección por CMV de localización no definida, las que fueron tratadas oportunamente. Se inició TARV con zidovudina (AZT), lamivudina (3TC), atazanavir/ritonavir (ATV/r) en mayo de 2009 contando en ese momento con un recuento de linfocitos T CD4 de 48 céls/mL y una carga viral de 136.000 copias ARN/mL. Posterior a ello no se controló pero retiró mensualmente su medicación. Siete meses después de iniciada la TARV desarrolló un síndrome confusional por lo que fue hospitalizado en noviembre de 2009. En su examen físico de ingreso destacaba una hemodinamia estable, ausencia de fiebre y presencia de algorra. Se lo describió con desorientación temporo-espacial, alteración de memoria a corto y largo plazo y en ocasiones soporoso. No presentaba déficit motor ni sensitivo. El hemograma y su bioquímica sanguínea no demostraron alteraciones clínicamente significativas. Los siguientes exámenes: HBsAg, anticuerpos anti VHC, IgG e IgM anti T. gondii, anticuerpos anti T. cruzi, y antigenemia para CMV fueron informados como negativos. El VDRL en sangre fue no reactivo. La TAC cerebral no demostró alteraciones. En un LCR inicial hubo 7 céls $/ \mathrm{mm}^{3}$, glucosa $44 \mathrm{mg} / \mathrm{mL}$, proteínas de $0,35 \mathrm{mg} / \mathrm{dL}$ y adenosin deaminasa de 2,5 mg/dL. En la tinción de Gram directa del LCR no se

\author{
Complejo Asistencial Dr. Sótero \\ del Río, Santiago de Chile. \\ Unidad de Infectología \\ M. Lasso ha participado en \\ protocolos de investigación, ha \\ dado charlas y asistió a conferencias \\ médicas tanto nacionales como \\ internacionales auspiciado por \\ las compañías farmacéuticas \\ BristolMyersSquibb y Abbott. \\ I. Cerón no tiene conflictos de \\ interés. \\ Trabajo sin fuente de \\ financiamiento. \\ Recibido: 19 de abril de 2011 \\ Aceptado: 27 de diciembre de \\ 2011
}

Correspondencia a: Martin Lasso Barreto zoalde@yahoo.com 


\section{Caso Clínico}

observaron microorganismos, el cultivo, la baciloscopia y la tinción de tinta china fueron negativos. El VDRL en LCR también fue no reactivo. La evaluación por el oftalmólogo resultó normal. La RM de cerebro demostró extensas lesiones de la sustancia blanca supratentorial y pedúnculos cerebrales (Figura $1 \mathrm{~A}$ ). Se le practicó una nueva punción lumbar para realizar RPC en LCR (anidada) para virus JC con resultado positivo que confirmó el diagnóstico de LEMP. Durante la evolución intra-hospitalaria se asistió a su deterioro neurológico con disfasia progresiva, imposibilidad para caminar y grave trastorno deglutorio lo que obligó a realizar una gastrostomía. Se decidió entonces modificar su TARV cambiando el ATV/r por lopinavir/ritonavir (LPV/r) y se mantuvieron AZT y 3TC. Además se inició mirtazapina 15 mg/día por sonda nasogástrica, tres semanas después de haber ingresado. Permaneció 48 días hospitalizado y al momento del alta en enero de 2010 estaba emaciado, vigil aunque no conectado con su entorno, no respondía órdenes simples, no emitía lenguaje, su alimentación era exclusivamente por gastrostomía y era incapaz de caminar. Quedó al cuidado de su madre y su hermana. Dos meses después de su alta estaba más reactivo con el medio y obedecía órdenes simples; su recuento de linfocitos CD4 era de 210 céls/mL y tenía carga viral indetectable. Cinco meses después del alta el paciente podía caminar, inició la alimentación por boca sin problema, aunque seguía usando gastrostomía, y estaba verborreico con lenguaje ininteligible; fue descrito como un niño de 3 a 4 años de edad. A los 7 meses post alta su lenguaje fue más claro y a los 10 meses ya no usaba su gastrostomía, se alimentaba enteramente por boca y sin restricción con el tipo de alimento. En el último control al cabo de un año de egresar del hospital, caminaba independientemente, se vestía, aseaba y alimentaba solo y su lenguaje era normal aunque se mostraba repetitivo de algunas palabras. En ese momento tenía un recuento de linfocitos T CD4 de 312 (7\%) céls/mL y su carga viral persistía indetectable al igual que en todos sus controles previos (4 en total). La RM cerebral de control (Figura 1B) fue realizada ocho meses tras el alta demostrando una marcada regresión del compromiso en la sustancia blanca, con elementos de atrofia cerebral.
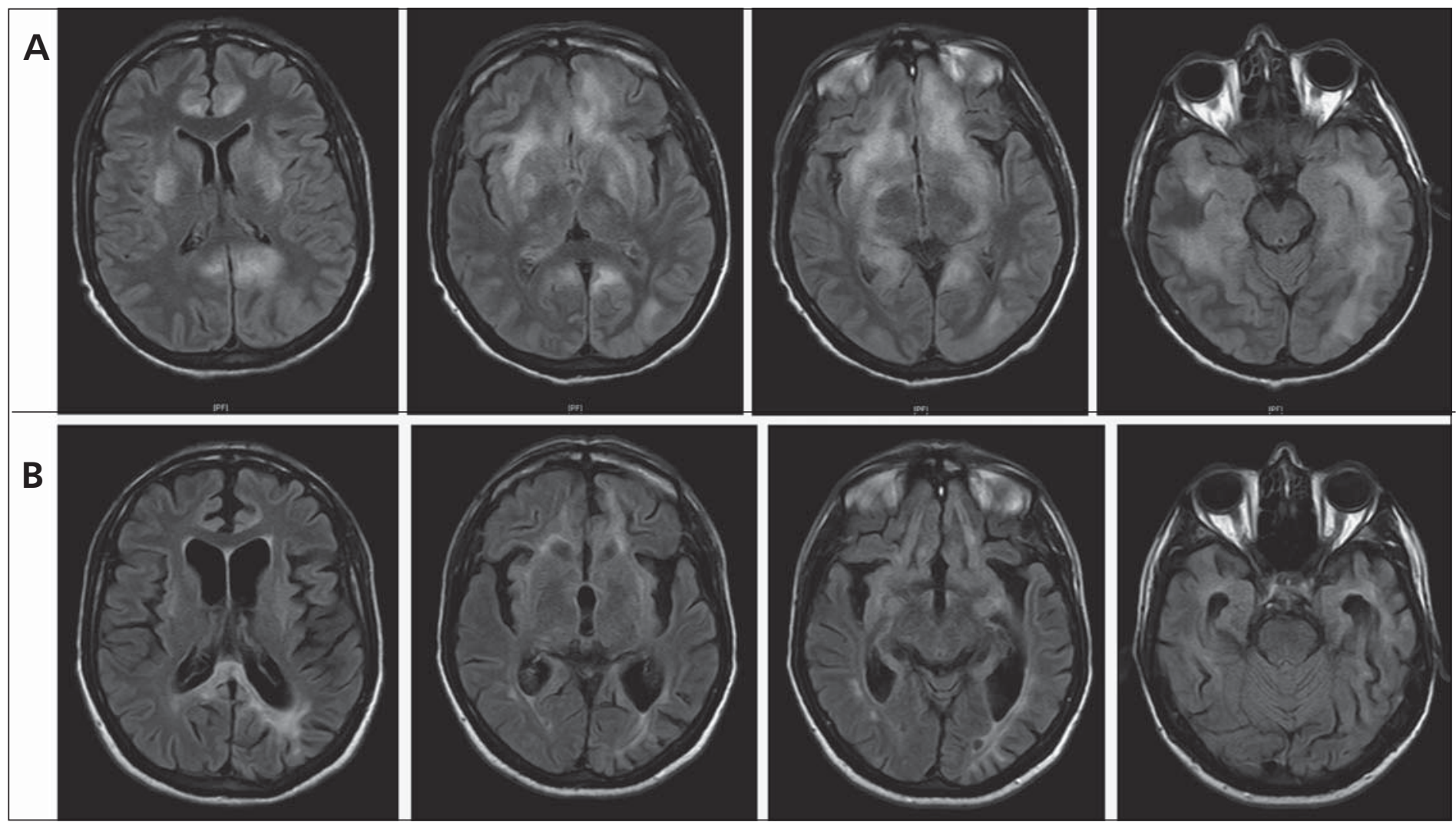

Figura 1. A: Focos de hiperseñal que compromete la sustancia blanca supratentorial de circunvoluciones rectas, orbitarias, circunvoluciones hipocampales, yuxta-articular tecal y frontal mesial y occipital bilateral, lóbulos temporales, núcleos lenticulares, esplenio del cuerpo calloso y de ambos pedúnculos cerebrales medios, visible en secuencias de TR largo, hipointensos en secuencias de T R corto. Con uso de contraste endovenoso no se observan refuerzos anormales (Fecha de examen 31/12/2009). B: Marcada regresión de los focos de hiperseñal que compromete la sustancia blanca supratentorial, observando solamente alteración de la señal en la región occipital bilateral, mayor a izquierda. Aumento del espacio subaracnoideo compatible con mayor atrofia cerebral en especial a nivel supratentorial (Fecha de examen 24/09/10). 


\section{Discusión}

La LEMP ocurre en el paciente infectado por VIH como una IO en condiciones de grave inmunosupresión, habitualmente con recuento de linfocitos T CD4 inferior a 100 céls/mL, aunque no es inhabitual encontrarla en pacientes con valores de linfocitos T CD4 superior a 100 e inferior 200 céls $/ \mathrm{mL}^{4}$. Se caracteriza por destrucción de la sustancia blanca cerebral y se manifiesta con déficit neurológico variable y asimétrico, destacando la presencia de hemiparesia (42\%), alteraciones cognitivas (36\%), defectos del campo visual (32\%), ataxia (21\%), afasia (17\%), déficit de pares craneales (13\%) y déficit sensitivos $(9 \%)^{5}$. Las lesiones en la LEMP se expanden de manera concéntrica o a través de los tractos de sustancia blanca, en el transcurso de semanas, nunca son de presentación abrupta, no se acompañan de cefalea ni fiebre y cerca de $20 \%$ desarrolla convulsiones, las que ocurren cuando las lesiones de sustancia blanca comprometen áreas inmediatamente adyacentes a la corteza cerebral ${ }^{6}$. La TAC cerebral demuestra zonas hipodensas, habitualmente periventriculares, asimétricas, que no siguen el patrón de territorio vascular, no producen efecto de masa ni captan los medios de contraste en 85 a 90\% de las veces, salvo que se encuentre en el contexto de un síndrome de reconstitución inmune donde, además de captar el contraste, puede existir edema perilesional con efecto de masa ${ }^{7}$ La RM cerebral es el examen de imagen de elección y demuestra lesiones en la sustancia blanca hiperintensas en T2 y FLAIR e hipointensas en $\mathrm{T} 1^{8}$. Debe realizarse una RPC para virus JC en el LCR para confirmar el diagnóstico, reacción que fuera evaluada en la era pre TARV con una sensibilidad de 60 a $90 \%{ }^{9,10}$ y una especificidad de 92 a $100 \%{ }^{11}$.

En nuestro paciente se cumplieron con las características clínicas, de imagen y microbiológicas-moleculares para tener un diagnóstico positivo de LEMP. La biopsia cerebral puede ser necesaria para establecer el diagnóstico de LEMP cuando la carga viral del virus JC es muy baja para ser detectada mediante RPC en el LCR ${ }^{12}$. El estudio histológico demuestra desmielinización multifocal, astrocitosis reactiva y oligodendrocitos con núcleos voluminosos e hipercromáticos en los que se detecta por inmunohistoquímica e hibridación in situ la presencia del virus JC, otorgando por esta vía el diagnóstico de certeza de LEMP5

Aunque al momento no existe tratamiento específico efectivo para la LEMP, sí se ha establecido beneficio en la sobrevida de pacientes con infección por VIH con LEMP que reciben TARV con buena penetración al SNC. Gasnault y cols. ${ }^{13}$, demostraron, en una gran cohorte francesa de 1.427 pacientes con LEMP reclutados y seguidos entre 1992 y 2004, una mortalidad de 58 por 100 personas-año en quienes no recibieron TAR, de 49 por 100 personas-año en aquellos que recibían TARV con baja penetración al SNC y una mortalidad 8 por 100 pacientes-año en aquellos con TARV de buena penetración al SNC. En la Tabla 1 se establecen los diversos grados de penetración al SNC de los fármacos anti-retrovirales ${ }^{14}$, destacando en particular zidovudina, nevirapina, abacavir, emtricitabina, efavirenz, darunavir/r, fosamprenavir/r, lopinavir/r, maraviroc y raltegravir como fármacos de paso fácil por la barrera hemato-encefálica y que están disponibles en el arsenal terapéutico de nuestro país. Independiente de aquello, son anecdóticas las publicaciones respecto de mejoría clínica neurológica significativa de LEMP exclusivamente con TARV ${ }^{15-17}$.

Para entender el probable rol de mirtazapina en el manejo de la LEMP, debemos comprender su patogenia recordando que la infección por el virus JC ocurre principalmente en la infancia; el virus permanece en condición latente en el tejido ganglionar y probablemente renal; luego, ante un evento mantenido de inmunosupresión y por vía de linfocitos B penetra al SNC; una vez allí infecta astrocitos y oligodendrocitos, estos últimos productores de mielina ${ }^{18}$. La afinidad tisular específica del virus JC se ve facilitada por un ligando específico dependiente de clatrina, proteína encargada de formar vesículas transportadoras. Se ha demostrado que fármacos inhibidores de dopamina y serotonina como son clorpromazina y clozapina bloquean la endocitosis dependiente de clatrina ${ }^{18,19}$. Por otro lado, debido a que las células gliales expresan receptores, tanto de dopamina como serotonina, existe la hipótesis de que el virus JC utiliza estos receptores para infectar el sistema glial y se ha demostrado in vitro que al bloquear los receptores serotoninérgicos mediante fármacos y anticuerpos monoclonales específicos, la replicación del virus JC desciende a niveles mínimos ${ }^{18}$. En este contexto se plantea el uso de mirtazapina, un antidepresivo con capacidad de ocupar los receptores presinápticos alfa 2 y los receptores de serotonina $5 \mathrm{HT}_{2 \mathrm{a}}$ impidiendo con ello el ingreso del virus JC. Existen publicaciones anecdóticas respecto del beneficio de este fármaco, tanto en pacientes infectados por $\mathrm{VIH}^{20,21}$ como en pacientes con LEMP demostrada sin co-infección por $\mathrm{VIH}^{22-24}$. Por otro lado,

\begin{tabular}{|c|c|c|c|c|}
\hline Anti-retroviral & 4 & 3 & 2 & 1 \\
\hline INTR* & Zidovudina & $\begin{array}{l}\text { Abacavir } \\
\text { Emtricitabina }\end{array}$ & $\begin{array}{l}\text { Didanosina } \\
\text { Lamivudina } \\
\text { Estavudina }\end{array}$ & $\begin{array}{l}\text { Tenofovir } \\
\text { Zalcitabina }\end{array}$ \\
\hline INNTR** $^{*}$ & Nevirapina & $\begin{array}{l}\text { Delaverdine } \\
\text { Efavirenz }\end{array}$ & Etravirina & \\
\hline $\mid \mathrm{P} * * *$ & Indinavir/r**** & $\begin{array}{l}\text { Darunavir/r } \\
\text { Fosamprenavir/r } \\
\text { Indinavir } \\
\text { Lopinavir/r }\end{array}$ & $\begin{array}{l}\text { Atazanavir } \\
\text { Atazanavir/r } \\
\text { Fosamprenavir }\end{array}$ & $\begin{array}{l}\text { Nelfinavir } \\
\text { Ritonavir } \\
\text { Saquinavir } \\
\text { Saquinavir/r } \\
\text { Tipranavir/r }\end{array}$ \\
\hline Inhibidores de entrada y fusión & & Maraviroc & & Enfuvirtide \\
\hline Inhibidores de integrasa & & Raltegravir & & \\
\hline \multicolumn{5}{|c|}{$\begin{array}{l}\text { *Inhibidores nucleósidos/nucleótidos de la transcriptasa reversa, }{ }^{* *} \text { Inhibidores no nucleósidos de la } \\
\text { transcriptasa reversa, }{ }^{* *} \text { inhibidores de proteasa, }{ }^{* * *} \text { ritonavir. De acuerdo al esquema que recibe e } \\
\text { paciente se suma el puntaje correspondiente a cada anti-retroviral y si éste es } \geq 9 \text { se considera de alta } \\
\text { eficacia con posibilidad de detectar } \mathrm{VIH}-1 \text { en } \mathrm{LCR} \text { de }<9 \% \text {. }\end{array}$} \\
\hline
\end{tabular}


también hay réplicas a esta teoría con estudios in-vitro que han demostrando presencia de virus JC en el endotelio microvascular cerebral independiente de los receptores serotoninérgicos ${ }^{25} \mathrm{y}$ la ausencia de efecto de risperidona (otro bloqueador $5 \mathrm{HT}_{2 \mathrm{a}}$ ) para impedir la infección de células gliales fetales humanas por el virus $\mathrm{JC}^{26}$.

La favorable evolución de nuestro paciente, sin embargo, nos abre a plantear el real beneficio de este medicamento siendo difícil separar cuánto de la mejoría pudo ser consecuencia de la optimización de la TARV y cuánto de la adición de mirtazapina y representa una invitación para profundizar en su eventual efecto terapéutico en la LEMP con estudios controlados prospectivos.

\section{Resumen}

Se presenta el caso clínico de un paciente de sexo masculino, de 43 años portador de VIH que desarrolló un grave daño neurológico subagudo debido a una leucoencefalopatía multifocal progresiva diagnosticada mediante reacción de polimerasa en cadena de virus JC. El paciente fue tratado con terapia anti-retroviral de penetración eficiente al SNC y con mirtazapina, un antidepresivo inhibidor de los receptores de serotonina. Su evolución durante un año de seguimiento ha sido favorable tanto del punto de vista clínico como de imágenes.

\section{Referencias}

1.- Padgett B L, Walker D L, Zurhein G M, Eckroade R J, Dessel B H. Cultivation of papova-like virus from human brain with progressive multifocal leukoencephalopathy. The Lancet 1971; 1 (7712): 1257-60.

2.- Khanna N, Elzi L, Mueller N J, Garzoni C, Cavassini M, Fux C A, et al. Incidence and outcome of progressive multifocal leukoencephalopathy over 20 years of the Swiss HIV Cohort Study. Clin Infect Dis 2009; 48: 1459-66.

3.- Antiretroviral Therapy Cohort Collaboration (ART-CC). Variable impact on mortality of AIDS-defining events diagnosed during combination antiretroviral therapy: Not all AIDS-defining conditions are created equal. Clin Infect Dis 2009; 48: 1138-51

4.- Gasnault J, Kahraman M, de Goër M G, Durali D, Delfraissy J F, Taufik Y. Critical role of JC virus-specific CD4 T cell responses in preventing progressive multifocal leukoencephalopathy. AIDS 2003; 17: 1443-9.

5.- Tan C S, Koralnik I J. Chapter 145: JC virus, BK and another polyomavirus: Progressive multifocal leukoencephalopathy. En Mandell, Douglas and Bennett's Principles and Practice of Infectious Diseases. Mandell, GL, Bennett JE, Dolin R editors. $7^{\text {th }}$ Edition. Philadelphia PA Churchill-Livingston-Elsevier. 2010 p : 2051-8.

6.- Lima M A, Drislane F W, Koralnik I J. Seizures and their outcome in progressive multifocal leukoencephalopathy. Neurology 2006; 66 (2): 262-4.

7.- Travis J, Varma A, duPlessis D, Turnbull I, Vilar F J. Immune reconstitution associated with progressive leukoencephalopthy in human immunodeficiency virus: a case discussion and review of the literature. Neurologist 2008; 14 (5): 321-6.

8.- Miranda G, Díaz C, Dellien H, Hermosilla H. Enfrentamiento imagenológico de las lesiones cerebrales en pacientes VIH. Rev Chil Radiol 2008; 14 (4): 200-7.

9.- Knowles W A, Pipkin P, Andrews N, Vyse A, Minor P, Brown D W, et al. Population-based study of antibody to the human polyomaviruses $\mathrm{BKV}$ and JCV and the simian polyomavirus
SV40. J Med Virol 2003; 71 (1): 115-23.

10.- Koralnik I J, Boden D, Mai V, Lord C, Letvin N. JC virus DNA load in patients with and without progressive multifocal leukoencephalopathy. Neurology 1999; 52 (2): 253-60.

11.- Cinque P, Scarpellini P, Vago L, Linde A, Lazzarin A. Diagnosis of central nervous system complications in HIV-infected patients: cerebrospinal fluid analysis by the polymerase chain reaction. AIDS 1997; 11 (1): 1-17.

12.- Cinque P, Koralnik IJ, Clifford D. The evolving face of progressive multifocal leukoencephalopathy: towards the definition of a consensus terminology. J Neurovirol 2003; 9: 88-92.

13.- Gasnault J, Lanoy E, Bentata M, Guiguet M, Costagliola D. Intracerebral penetrating ART are more efficient on survival HIV + patients with progressive multifocal leucoencephalopathy (ANRS CO4-FHDH) $15^{\text {th }}$ Conference on Retroviruses and Opportunistic Infections. Boston 2008 Abstract 385.

14.- Letendre S, FitzSimons C, Ellis R, Clifford D, Collier A, Gelman B, et al. Correlates of CSF viral loads in 1221 volunteers of the CHARTER cohort. $17^{\text {th }}$ Conference on Retroviruses and Opportunistic Infections. San Francisco 2010 Abstract 172.

15.- Albrecht H, Hoffmann C, Degen O, Stoehr A, Plettenberg A, Mertenskötter T, et al. Highly active antiretroviral therapy significantly improves the prognosis of patients with HIV-associated progressive multifocal leukoencephalopathy. AIDS 1998; 12 (10): 1149.

16.- Elliot B, Aromin I, Gold R, Flanigan T, Mileno M. 2.5 year remission of AIDSassociated progressive multifocal leukoencephalopathy with combined antiretroviral therapy. Lancet 1997; 349 (9055): 850.

17.- Turnher M, Donovan M J, Rieger A, Kleibl-Popov C, Loewe C, Schindler E. Initial and follow-up MR imaging findings in AIDS related progressive multifocal leukoencephalopathy treated with highly active antiretroviral therapy. AJNR 2001; 22: 977-84.
18.- Elphick G F, Querbes W, Jordan J A, Gee G V, Eash S, Manley K, et al. The human polyomavirus. Uses serotonin receptors to infect cells. Science 2004; 306 (5700): 1380-3.

19.- Baum S, Ashok A, Gee G, Dimitrova S, Querbes W, Jordan J, et al. Early events in the life cycle of JC virus as potential therapeutic targets for the treatment of progressive multifocal leukoencephalopathy. J Neurovirol 2003; 9 (Suppl1): S32-S37.

20.- Cettomai D, McArthur J C. Mirtazapine use in human immunodeficiency virusinfected patients with progressive multifocal leukoencephalopathy. Arch Neurol 2009; 66 (2): 255-8.

21.- Lanzafame M, Ferrari S, Lattuada M, Corsini F, Deganello R, Vento S, et al. Mirtazapine in an HIV-1 infected patient with progressive multifocal leukoencephalopathy. Infez Med 2009; 17 (1): 35-7.

22.- Vulliemoz S, Lurati-Ruiz F, Borruat F X, Delavelle J, Koralnik I, Kuntzer T, et al. J Favourable outcome of progressive multifocal leucoencephalopathy in two patients with dermatomyositis. Neurol Neurosurg Psychiatry 2006; 77 (9): 1079-82.

23.- Owczarczyk K, Hilker R, Brunn A, Hallek M, Rubbert A. Progressive multifocal leucoencephalopathy in a patient with sarcoidosis-successful treatment with cidofovir and mirtazapine. Reumatology (Oxford) 2007; 46 (5): 888-90.

24.- Verma S, Cikurel K, Koralnik I, Morgello S, Cunningham-Rundles C, Weinstein Z. Mirtazapine in progressive multifocal leukoencephalopathy associated with polycythemia vera. J Infect Dis 2007; 196 (5): 709-11.

25.- Chapagain M L, Verma S, Mercier F, Yanagihara R, Nerurkar V R. Polyomavirus JC infects human brain microvascular endotelial cells independent of serotonin receptor 2. Virology 2007; 364 (1): 55-63

26.- Chapagain M L, Sumibcay L Gurjav U, Kaufusi P H, Kast R E, Nerurkar V R. Serotonin receptor 2A blocker (risperidone) has no effect on human polyomavirus JC infection of primary human fetal glial cells. J NeuroVirol 2008; 14 : 448-54. 